\title{
Importance of the immune response to Mycobacterium leprae in the skin
}

\author{
Song-Hyo Jin ${ }^{1}$, Kyu Joong $\mathrm{Ahn}^{2}$ and Sungkwan $\mathrm{An}^{3^{*}}$ (D)
}

\begin{abstract}
The causative agent of leprosy is Mycobacterium leprae (M. leprae), which establishes infectious lesions in the skin. Leprosy is classified based on the clinical manifestation, the host's immune response and skin symptoms. M. leprae is an intracellular pathogen that invades keratinocytes, macrophages, dendritic cells and Schwann cells and replicates within these cells. M. leprae-infected keratinocytes secrete various cytokines and chemokines and induce highly effective immune responses. Understanding the mechanisms by which $M$. leprae establishes an infection within the skin and the associated immune response may be of great help in the early detection and treatment of the disease.
\end{abstract}

Keywords: Mycobacterium leprae, Leprosy, Immune response, Keratinocytes

\section{Background}

Leprosy is a chronic granulomatous infection caused by an intracellular organism, Mycobacterium leprae (M. leprae), which primarily affects the skin and peripheral nerves (Walker and Lockwood 2006). M. leprae is a unique type of bacteria as it has a long generation time and does not grow on an artificial medium. In addition, $M$. leprae is $0.3-7.0 \mu \mathrm{m}$ in size and is an exclusively intracellular parasite that grows extremely slowly with a generation time of 12-14 days (Sasaki et al. 2001). The most striking feature of the $M$. leprae genome is the extensive deletion and inactivation of genes, referred to as gene degradation (Cole et al. 2001). These characteristics of the M. leprae genome can explain its slow growth and failure to proliferate in synthetic media. The human genomes PARK2 and PACRG are associated with increased susceptibility to leprosy or more severe forms of leprosy.

The host's immune system affects the clinical manifestation of leprosy. Strong cell-mediated immunity and low humoral immunity characterise the response to tuberculoid (TT) leprosy, whereas in lepromatous (LL) leprosy, the opposite is observed. Leprosy can be classified more precisely in an immunological context based on skin findings, motor and sensory changes and biopsy findings as indeterminate (I), TT, borderline tuberculoid (BT), mid-borderline (BB), borderline lepromatous (BL) and LL (Jacobson and Krahenbuhl 1999). In addition, $M$. leprae invades and survives within macrophages, dendritic cells and Schwann cells. Interleukin 2 (IL-2) and interferon gamma (IFN- $\gamma$ ) are markedly dominant in TT lesions, whereas IL-4, IL-5 and IL-10 are characteristic of LL lesions (Salgame et al. 1991; Yamamura et al. 1991).

Leprosy is a disease that manifests as skin lesions, and keratinocytes and the epidermis play an important role in the innate immune response to $M$. leprae (Lyrio et al. 2015). Moreover, while some evidence indicates a role for dendritic cells (DCs) in the immune response to M. leprae (Santos et al. 2001), another cell type important for epidermal defence, the keratinocyte, is also a source of cytokines and chemokines, which are critical for recruiting DCs, T cells and neutrophils to the site of infection (Lyrio et al. 2015). In addition, human keratinocytes have been shown to phagocytose $M$. leprae in vitro and subsequently exhibit changes in the expression of surface molecules and cathelicidin as well as secrete tumour necrosis factor (TNF)- $\alpha$ and IL-1 $\beta$ (Lyrio et al. 2015). In addition, the invasion of keratinocytes and the secretion of cytokines and chemokines by immune cells were reported. This suggests that keratinocytes play an important role in the immune response to an infection with $M$. leprae.

\footnotetext{
* Correspondence: ansungkwan@konkuk.ac.kr

${ }^{3}$ Department of Cosmetics Engineering, Konkuk University, 120

Neungdong-ro, Gwangjin-gu, Seoul 05029, Republic of Korea

Full list of author information is available at the end of the article
}

(c) The Author(s). 2018 Open Access This article is distributed under the terms of the Creative Commons Attribution 4.0 International License (http://creativecommons.org/licenses/by/4.0/), which permits unrestricted use, distribution, and reproduction in any medium, provided you give appropriate credit to the original author(s) and the source, provide a link to the Creative Commons license, and indicate if changes were made. The Creative Commons Public Domain Dedication waiver (http://creativecommons.org/publicdomain/zero/1.0/) applies to the data made available in this article, unless otherwise stated. 


\section{Pathophysiology of leprosy}

$M$. leprae is an intracellular parasitic pathogen, and attempts to cultivate in artificial medium have failed since 1874 when it was first identified by Armauer Hansen (Walker and Lockwood 2006). In addition, it multiplies extensively in the footpads of nude mice (Shepard 1960), nine-banded armadillos (Kirchheimer and Storrs 1971) and, to a limited extent, in the footpads of normal mice (Sasaki et al. 2001). M. leprae bacilli are $0.3-0.4 \times 4.0-7.0 \mu \mathrm{m}$ in size and multiplies very slowly, with a generation time of 12-14 days. Optimal growth occurs at approximately $30{ }^{\circ} \mathrm{C}$; hence, $M$. leprae prefers the cooler areas of the human body. The cell wall is gram-positive and highly complex and contains proteins, phenolic glycolipids, arabinoglycan, peptidoglycan and mycolic acid (Sasaki et al. 2001).

The host's immune system affects the clinical manifestation of leprosy. Strong cell-mediated immunity and low humoral immunity characterises the response to TT leprosy, whereas the opposite is observed in cases of LL leprosy (Jacobson and Krahenbuhl 1999).

The most striking feature of the $M$. leprae genome is the extensive deletion and inactivation of genes, referred to as gene degradation; only $49.5 \%$ of the genome contains protein-coding genes and $27 \%$ contains recognisable pseudogenes (inactive reading frames with functional counterparts in the tuberculosis bacillus). Moreover, an analysis of the genomic sequence revealed that the genes encoding various enzymes have been replaced by pseudogenes, which suggests limited metabolic activity of $M$. leprae (Cole et al. 2001). This genomic feature might correspond to its unique bacteriological characteristics, including its exceptionally slow growth rate and failure to multiply in synthetic media (Sasaki et al. 2001).

There are various genes and regions of the human genome that have been associated with susceptibility to leprosy or more severe forms of the disease (Walker and Lockwood 2006). For example, Mira et al. (Mira et al. 2004) identified certain alleles in the PARK2 and PACRG region of chromosome 6 to be associated with susceptibility to leprosy in Vietnamese and Brazilian cohorts. Moreover, PARK2 is expressed by both Schwann cells and macrophages. It is an ubiquitination E3 ligase involved in the delivery of polyubiquitinated proteins to the proteasome complex for protein degradation (Ciechanover 2006). An Indian cohort demonstrated that homozygotes expressing different alleles of the vitamin $\mathrm{D}$ receptor (VDR) gene were associated with either TT or LL leprosy (Roy et al. 1999). In addition, the upregulation of the VDR gene on macrophages is associated with enhanced intracellular killing of M. tuberculosis (Liu et al. 2006).

\section{Classification of leprosy}

Leprosy is classified according to the WHO guidelines (World Health Organization 2012). Patients with only one skin lesion are categorised as a single lesion paucibacillary; however, paucibacillary leprosy is defined as five or fewer skin lesions without bacilli in the skin smears. Multibacillary denotes more than six lesions and may be skin smear positive. TT leprosy is characterised by a minor loss of nerve, the presence of few bacilli and strong cell-mediated immunity (i.e. IFN- $\gamma$ and IL-2) and weak humoral immunity. In contrast, LL leprosy induces strong bacterial immunity (i.e. IFN-beta, IL-4 and IL-10) and cell-mediated immunity exhibited by a wide range of lesions, multiple bacteria as well as lesions with extensive skin and nerve involvement.

\section{TT}

In TT, patients have one or two larger macular hypopigmented or erythematous anaesthetic lesions that have a well-defined and often raised margin or appear as scaly plaques (Jacobson and Krahenbuhl 1999). The first type of lesion is a macule that has erythema or hypochloremesis and has a dry, hairless surface and a well-defined outer edge and sensory damage. Foci of well-developed epithelioid cells, with or without Langhans giant cells, are encompassed by a zone of dense lymphocyte infiltration. The granuloma, which extends up to the epidermis, is without an intervening clear zone (Ridley and Jopling 1966).

\section{BT}

In BT, the macules or plaques resemble $\mathrm{TT}$ leprosy in appearance and sensory loss but can be differentiated by the fact that they have a smaller average size, are more numerous, the surface is less dry, the outer edges are less defined, hair growth is less affected and nerves are thickened. The cytology and composition of the granuloma are typically indistinguishable from those of TT. The most distinguishing characteristic is the presence of a clear subepidermal zone; however, it is very narrow. Moreover, the granulomas can be distinguished from $\mathrm{BB}$ based on epithelial cell focalization near the peripheral lymphocyte region or occasionally by the presence of Langhans giant cells. The nerve bundles within the granuloma are generally grossly swollen and infiltrated, and innervation is greatly diminished (Ridley and Jopling 1966).

\section{BB}

In cases of $\mathrm{BB}$, the lesion size and number is between that of TT and LL, moderate anaesthesia and exhibits a typical 'punched-out' or 'hole-in-cheese' appearance. The essential defining characteristic is the presence of epithelial cells diffused throughout the granuloma and not by the lymphocyte zone. The epithelial cells are welldeveloped but generally not as large as those in TT leprosy. BB lesions contain no Langhans giant cells, and if 
lymphocytes are present, they are highly diffuse. In addition, the nerve bundles exhibit moderate Schwann cell proliferation but they are usually recognisable without much difficulty (Ridley and Jopling 1966).

\section{BL}

BL lesions tend to be numerous and particularly macular and consist of lacerations, papules and nodules. There are two types of BL leprosy: (1) granulomas that consist of histiocytic cells that cannot be classified as epithelial cells but tend to evolve into epithelial cells and (2) the $M$. leprae host cells that consist of histiocytes that tend to exhibit foamy changes; however, they do not produce large globes. The granulomas can be distinguished from LL granulomas by the presence of dense lymphocytic infiltration (Ridley and Jopling 1966).

\section{LL}

LL lesions typically consist of erythematous macules, papules and/or nodules, which are widespread and can occasionally become diffuse without defined lesions. In addition, the lesions may appear similar to TT but with more BT and BL characteristics. Sensory and/or motor loss usually occurs in the nerves near TT lesions but may be more prevalent in BL and LL leprosy. In addition, nerve damage is a common form of sensory loss and occurs at the final stages of LL leprosy. The ulnar and median (clawed hands), the common peroneal (foot drop), the posterior tibial (claw toes and plantar insensitivity) and the facial, radial cutaneous and great auricular nerves are involved. Occasionally, progressive multibacillary LL leprosy can result in the loss of the eyebrows and eyelashes, nasal septal perforation with a collapsed nose and hoarseness (Jacobson and Krahenbuhl 1999). Moreover, the granuloma is composed of histiocytes that exhibit a varying degree of fatty changes, characterised by the production of foam cells and globi. Numerous globi or heavy foamy changes are only found in LL leprosy. Lymphocytes are usually deficient and diffuse if they are present. Nerves can show structural damage but do not exhibit cell penetration or cuffing (Ridley and Jopling 1966).

\section{Immunology of leprosy}

Leprosy exhibits a wide variety of clinical features that are dependent on the host's immune response and has an apparent polarity in the form of TT and LL leprosy. The major defence against $M$. leprae is achieved by cellmediated immunity, and the outcome of the infection depends on how the host responds to the infection (Modlin 1994).

M. leprae invades and survives within macrophages, DCs and Schwann cells. Entry into the host cell is the first step in the intracellular lifecycle of M. leprae, which is achieved via several different methods. In particular, the phenolic glycolipid 1 (PGL 1) expressed on the cell wall of $M$. leprae is recognised by complement, and the complement receptor (CR) 1, CR 3 and CR 4 assist in phagocytosis of the bacilli (Schlesinger and Horwitz 1991). Host cells recognise many pathogens through general molecular pattern recognition; however, the complement and tolllike receptors (TLRs) expressed on macrophages and DCs are important for the recognition of microbial, including mycobacterial, pathogens (Nath et al. 2015).

IL- 2 and IFN- $\gamma$ are markedly dominant in TT lesions, whereas IL-4, IL-5 and IL-I0 are characteristic of LL lesions (Salgame et al. 1991; Yamamura et al. 1991). Moreover, the $\mathrm{T}$ helper type 1 (Th1) subset, characterised by the predominant secretion of IL- 2 and IFN- $\gamma$, preferentially elicits cell-mediated immunity, whereas Th2 cells, which produce IL-4, IL-5 and IL-10, augment the humoral immune response. Both the classic reciprocal relationship between antibody production and cellmediated immunity and the resistance or susceptibility to $M$. leprae can be explained by $\mathrm{T}$ cell subsets differing in the pattern of cytokine production (Sasaki et al. 2001). The mechanism of $T$ cell activation in response to mycobacteria is highlighted by the CD1-mediated lipid antigen presentation pathway (Moody et al. 1996) as it represents an aspect of host defence independent of classical peptide antigen presentation via major histocompatibility complex (MHC) molecules (Sasaki et al. 2001).

M. leprae activates TLR2 and TLR1 in Schwann cells, which specifically leads to TT leprosy. Although this cell-mediated immune response is most active in TT leprosy, it can also activate apoptosis genes and consequently cause nerve damage in cases of TT leprosy. In addition, the alpha-2 laminin receptors found in the basal lamina of Schwann cells are also an entry target for $M$. leprae in these cells, while the activation of the ErbB2 receptor tyrosine kinase signalling pathway has been identified as a mediator of demyelination in leprosy (Tapinos et al. 2006). The activation of macrophages and DCs, which are antigen-presenting cells, is associated with the initiation of the host immune response to $M$ leprae. Moreover, IL-1 $\beta$ produced by antigen-presenting cells has been shown to impair the maturation and function of DCs (Makino et al. 2006). Another mechanism is the ubiquitin-proteasome pathway, which causes immune cell death and tumour necrosis factor (TNF)- $\alpha /$ IL-10 secretion (Fulco TO et al. 2007). Vitamin D can contribute to the intrinsic response through the production of antimicrobial peptides and is differentially expressed in TT leprosy compared to that in LL leprosy. On the other hand, IL-10 can induce phagocytosis. In addition, it has been shown that IL-15 induced the vitamin D antimicrobial pathway and decreased phagocytosis (Nath et al. 2015). While TT leprosy is the result of 
a high cell-mediated immunity with a Th1-type immune response, LL leprosy is characterised by a low level of cellular immunity due to an increased humoral Th2 response (Modlin 1994). Because M. leprae is present in the skin, nerve tissue and endothelium of the nasal mucosa, it is thought that endothelial cells contribute to the pathogenesis of leprosy.

\section{Keratinocytes and leprosy}

Keratinocytes express mannose-binding receptors (KCMR), TLRs and Class II MHC antigens and have also been identified as a source of cytokines, chemokines and antimicrobial peptides. In addition, keratinocytes may play an important role in leprosy by participating in the epidermal immune response to $M$. leprae (Mutis et al. 1993). Thus, this cell type possesses a highly sophisticated innate pattern recognition system in which the simultaneous recognition of a pathogen by different classes of pattern recognition receptors can provide a specific immune response or, in the case of commensals, a lack of a response to microorganisms (Lyrio et al. 2015). Furthermore, keratinocytes can distinguish between pathogenic and commensal microorganisms (Pivarcsi et al. 2005). In skin biopsy sections obtained from an LL leprosy patient, M. leprae were found in macrophages as well as in smooth muscle cells and keratinocytes, suggesting that the skin is a potential route of leprosy transmission (Satapathy et al. 2005). Keratinocytes also spontaneously express CD80 (B7-1) on their surface. A reduction of $\mathrm{CD} 80$ surface expression also occurs on monocytes after LPS exposure during hypoxia (Lahat et al. 2003). Moreover, Nickoloff et al. reported that keratinocytes can regulate $\mathrm{T}$ cells through both cytokine expression and CD80 and CD28 interactions (Nickoloff et al. 1995).

It has been established that epidermal keratinocytes express human beta-defensin (HBD) 2 and that this expression is upregulated by TNF- $\alpha$ stimulation (Yang et al. 1999). TNF- $\alpha$ induces MIP-3 $\alpha$ in human keratinocytes and recruits Langerhans cells to the epidermis (Tohyama et al. 2001). Additionally, TNF- $\alpha$ has a synergistic effect on Th1 pattern maintenance as IFN- $\gamma$ in synergy with TNF- $\alpha$ activates infected macrophages. As a result, it induces a major effect on cell-mediated immunity (Tohyama et al. 2001). Most recently, Cogen et al. have demonstrated that M. leprae induced HBD 2 and 3 in keratinocytes but not in macrophages (Cogen et al. 2012).

Okada et al. suggested that $M$. leprae can be phagocytosed by keratinocytes (Okada et al. 1978). Epidermal changes, Ia (HLA-DR) expression on keratinocytes, Langerhans cell (LC) hyperplasia and lymphocyte infiltration were identified in the skin lesions of leprosy patients (Rea et al. 1986). Seo et al. reported that the bacilli are located within typical epidermal cells, which exhibit tonofilaments and melanosomes in their cytoplasm as well as desmosomes at the junction of each of the cells (Seo et al. 1995). Human keratinocytes have been shown to phagocytose $M$. leprae in vitro, which induces changes in the expression of various surface molecules and cathelicidin as well as the secretion of TNF- $\alpha$ and IL-1 $\beta$ by these cells (Lyrio et al. 2015). Cytokines and chemokines derived from keratinocytes are important for the mobilisation of LCs, DCs, T cells and neutrophils to the site of infection, where they can mediate microbial killing and initiate a more efficient immune response against $M$. leprae.

\section{Conclusions}

Leprosy exhibits a wide variety of clinical features depending on the host's immune response and has an apparent polarity in the form of TT and LL leprosy. A majority of individuals do not develop leprosy or become infected following regular exposure. Those who have a latent $M$. leprae infection for years may have only single lesions, which is often selfhealing. If the lesion does not self-heal and the single lesion is not treated, the disease can progress to the paucibacillary or multibacillary stage (Jacobson and Krahenbuhl 1999).

Research to uncover new targets for the early detection and treatment of an $M$. leprae infection should continue to gain insight into the pathophysiology of leprosy. The most recent studies have included interferon (Teles et al. 2013), vitamin D-dependent antimicrobial pathways (Liu et al. 2012), NOD2-mediated signalling (Netea et al. 2010) and the role of $\mathrm{T}$ regulatory cells, Th-17/IL17a/IL-17F cytokines, CD163 and galectin-3 (Polycarpou et al. 2013). A deeper understanding of the $M$. leprae genome will provide insight into the mechanism by which this organism avoids immune surveillance.

$M$. leprae invades the skin and peripheral nerves, causing leprosy. Thus, the skin epithelial cells and keratinocytes are important in the innate immune response towards this bacterium. Because keratinocytes express mannose-binding receptors (KCMR), TLRs and Class II MHC antigens as well as produce cytokines, chemokines and antimicrobial peptides, they may play an important role and participate in the epidermal immune response to M. leprae (Mutis et al. 1993). It has been demonstrated in vitro that human keratinocytes can phagocytose $M$. leprae and subsequently exhibit the expression of the surface molecules CD80, CD209 and cathelicidin as well as secrete TNF- $\alpha$ and IL-1 $\beta$ (Lyrio et al. 2015). $M$. leprae induces the production of cytokines and chemokines in keratinocytes, which mediates mycobacterial killing and results in a more efficient immune response 
that is important for the recruitment of LCs, DCs, T cells and neutrophils. Because keratinocytes play an important role in the immune response against mycobacteria, we will present basic data for the early detection and treatment of leprosy by studying the interaction between keratinocytes and M. leprae.

\section{Abbreviations}

IFN-Y: Interferon gamma; BB: Mid-borderline; BL: Borderline lepromatous; BT: Borderline tuberculoid; CR: Complement receptor; DCs: Dendritic cells; HBD: Human beta-defensin; KCMR: Keratinocytes express mannose-binding receptors; LC: Langerhans cells; LL: Lepromatous; IL-2: Interleukin 2; M. leprae: Mycobacterium leprae; MHC: Major histocompatibility complex; PGL 1: Phenolic glycolipid 1; Th1: T helper type 1; TLRs: Toll-like receptors; TNF: Tumour necrosis factor; TT: Tuberculoid; VDR: Vitamin D receptor

\section{Acknowledgements}

Not applicable.

\section{Funding}

Not applicable.

\section{Availability of data and materials}

Not applicable.

\section{Authors' contributions}

SHJ, KJA and SA analysed the data, reviewed the literatures and wrote the manuscript. All authors read and approved the final manuscript.

\section{Ethics approval and consent to participate}

Not applicable.

\section{Consent for publication}

Not applicable.

\section{Competing interests}

The authors declare that they have no competing interests.

\section{Publisher's Note}

Springer Nature remains neutral with regard to jurisdictional claims in published maps and institutional affiliations.

\section{Author details}

'Department of Pathology College of Medicine, Institute of Hansen's Disease, The Catholic University of Korea, Seoul 06591, Republic of Korea. ${ }^{2}$ Department of Dermatology, Konkuk University School of Medicine, Seoul 05029, Republic of Korea. ${ }^{3}$ Department of Cosmetics Engineering, Konkuk University, 120 Neungdong-ro, Gwangjin-gu, Seoul 05029, Republic of Korea.

Received: 21 May 2017 Accepted: 19 December 2017

Published online: 20 January 2018

\section{References}

Ciechanover A. The ubiquitin proteolytic system: from a vague idea, through basic mechanisms, and onto human diseases and drug targeting. Neurology. 2006;66(Suppl 1):7-19.

Cogen AL, Walker SL, Roberts CH, Hagge DA, Neupane KD, Khadge S, et al. Human beta-defensin 3 is up-regulated in cutaneous leprosy type 1 reactions. PLoS Negl Trop Dis. 2012;6:e1869.

Cole ST, Eiglmeier K, Parkhill J, James KD, Thomson NR, Wheeler PR, et al. Massive gene decay in the leprosy bacillus. Nature. 2001;409:1007-11.

Fulco TO, Lopes UG, Sarno EN, Sampaio EP, Saliba AM. The proteasome function is required for mycobacterium leprae-induced apoptosis and cytokine secretion. Immunol Lett. 2007;110:82-5.

Jacobson RR, Krahenbuhl JL. Leprosy. Lancet. 1999;353:655-60.

Kirchheimer WF, Storrs EE. Attempts to establish the armadillo (Dasypus novemcinctus Linn.) as a model for the study of leprosy. I. Report of lepromatoid leprosy in an experimentally infected armadillo. Int J Lepr Other Mycobact Dis. 1971;39:693-702.
Lahat N, Rahat MA, Ballan M, Weiss-Cerem L, Engelmayer M, Bitterman H. Hypoxia reduces CD80 expression on monocytes but enhances their LPSstimulated TNF-alpha secretion. J Leukoc Biol. 2003;74:197-205.

Liu PT, Stenger S, Li H, Wenzel L, Tan BH, Krutzik SR, et al. Toll-like receptor triggering of a vitamin D-mediated human antimicrobial response. Science. 2006:311:1770-3.

Liu PT, Wheelwright M, Teles R, Komisopoulou E, Edfeldt K, Ferguson B, et al. MicroRNA-21 targets the vitamin D-dependent antimicrobial pathway in leprosy. Nat Med. 2012;18:267-73.

Lyrio EC, Campos-Souza IC, Corrêa LC, Lechuga GC, Verícimo M, Castro HC, et al. Interaction of Mycobacterium leprae with the $\mathrm{HaCaT}$ human keratinocyte cell line: new frontiers in the cellular immunology of leprosy. Exp Dermatol. 2015; 24:536-42.

Makino M, Maeda Y, Mukai T, Kaufmann SH. Impaired maturation and function of dendritic cells by mycobacteria through IL-1beta. Eur J Immunol. 2006;36:1443-52.

Mira MT, Alcaïs A, Nguyen VT, Moraes MO, Di Flumeri C, HT V, et al. Susceptibility to leprosy is associated with PARK2 and PACRG. Nature. 2004;427:636-40.

Modlin RL. Th1-Th2 paradigm: insights from leprosy. J Invest Dermatol. 1994;102: $828-32$.

Moody DB, Sugita M, Peters PJ, Brenner MB, Porcelli SA. The CD1-restricted T-cell response to mycobacteria. Res Immunol. 1996;147:550-9.

Mutis T, De Bueger M, Bakker A, Ottenhoff THHLA. Class II+ human keratinocytes present Mycobacterium leprae antigens to CD4+ Th1-like cells. Scand J Immunol. 1993;37:43-51.

Nath I, Saini C, Valluri VL. Immunology of leprosy and diagnostic challenges. Clin Dermatol. 2015;33:90-8.

Netea MG, Kullberg BJ, van der Meer JW. Genomewide association study of leprosy. N Engl J Med. 2010;362:1447-8.

Nickoloff BJ, Turka LA, Mitra RS, Nestle FO. Direct and indirect control of T-cell activation by keratinocytes. J Invest Dermatol. 1995;105(Suppl1):25-9.

Okada S, Komura J, Nishiura M. Mycobacterium leprae found in epidermal cells by electron microscopy. Int I Lepr Other Mycobact Dis. 1978;46:30-4.

Pivarcsi A, Nagy I, Kemeny L. Innate immunity in the skin: how keratinocytes fight against pathogens. Curr Immunol Rev. 2005;1:29-42.

Polycarpou A, Walker SL, Lockwood DN. New findings in the pathogenesis of leprosy and implications for the management of leprosy. Curr Opin Infect Dis. 2013;26:413-9.

Rea TH, Shen JY, Modlin RL. Epidermal keratinocyte la expression, Langerhans cell hyperplasia and lymphocytic infiltration in skin lesions of leprosy. Clin Exp Immunol. 1986;65:253-9.

Ridley DS, Jopling WH. Classification of leprosy according to immunity. A fivegroup system. Int J Lepr Other Mycobact Dis. 1966;34:255-73.

Roy S, Frodsham A, Saha B, Hazra SK, Mascie-Taylor CG, Hill AV. Association of vitamin D receptor genotype with leprosy type. J Infect Dis. 1999;179:187-91.

Salgame P, Abrams JS, Clayberger C, Goldstein H, Convit J, Modlin RL, et al. Differing lymphokine profiles of functional subsets of human CD4 and CD8 T cell clones. Science. 1991;254:279-82.

Santos DO, Santos SL, Esquenazi D, Nery JA, Defruyt M, Lorré K, et al. Evaluation of B7-1 (CD80) and B7-2 (CD86) costimulatory molecules and dendritic cells on the immune response in leprosy. Nihon Hansenbyo Gakkai Zasshi. 2001;70:15-24.

Sasaki S, Takeshita F, Okuda K, Ishii N. Mycobacterium leprae and leprosy: a compendium. Microbiol Immunol. 2001;45:729-36.

Satapathy J, Kar BR, Job CK. Presence of mycobacterium leprae in epidermal cells of lepromatous skin and its significance. Indian J Dermatol Venereol Leprol. 2005;71:267-9.

Schlesinger LS, Horwitz MA. Phenolic glycolipid-1 of Mycobacterium leprae binds complement component C3 in serum and mediates phagocytosis by human monocytes. J Exp Med. 1991;174:1031-8.

Seo VH, Cho W, Choi HY, Hah YM, Cho SN. Mycobacterium leprae in the epidermis: ultrastructural study I. Int I Lepr Other Mycobact Dis. 1995;63:101-4.

Shepard CC. The experimental disease that follows the injection of human leprosy bacilli into foot-pads of mice. J Exp Med. 1960;112:445-54.

Tapinos N, Ohnishi M, Rambukkana A. ErbB2 receptor tyrosine kinase signaling mediates early demyelination induced by leprosy bacilli. Nat Med. 2006;12:961-6.

Teles RM, Graeber TG, Krutzik SR, Montoya D, Schenk M, Lee DJ, et al. Type I interferon suppresses type II interferon-triggered human anti-mycobacterial responses. Science. 2013;339:1448-53.

Tohyama M, Shirakara Y, Yamasaki K, Sayama K, Hashimoto K. Differentiated keratinocytes are responsible for TNF-alpha regulated production of macrophage inflammatory protein 3alpha/CCL20, a potent chemokine for Langerhans cells. J Dermatol Sci. 2001;27:130-9. 
Walker SL, Lockwood DN. The clinical and immunological features of leprosy. Br Med Bull. 2006;77-78:103-21.

World Health Organization. WHO expert committee on leprosy. World Health Organ Tech Rep Ser. 2012;968:1-61.

Yamamura M, Uyemura K, Deans RJ, Weinberg K, Rea TH, Bloom BR, et al. Defining protective responses to pathogens: cytokine profiles in leprosy lesions. Science. 1991;254:277-9.

Yang D, Chertov O, Bykovskaia SN, Chen Q, Buffo MJ, Shogan J, et al. Betadefensins: linking innate and adaptive immunity through dendritic and $T$ cell CCR6. Science. 1999;286:525-8.

Submit your next manuscript to BioMed Central and we will help you at every step:

- We accept pre-submission inquiries

- Our selector tool helps you to find the most relevant journal

- We provide round the clock customer support

- Convenient online submission

- Thorough peer review

- Inclusion in PubMed and all major indexing services

- Maximum visibility for your research

Submit your manuscript at www.biomedcentral.com/submit
Biomed Central 